\title{
Structural Comparison of Scissor-hinge Linkages
}

\author{
F. Maden \\ Department of Architecture, Yaşar University, İzmir, Turkey \\ Y. Akgün \\ Department of Urban Design, Konak Municipality, Izmir, Turkey
}

K. Yücetürk \& E. Aktaş

Department of Civil Engineering, İzmir Institute of Technology, İzmir, Turkey

M.Y. Uncu

Department of Architecture, İzmir Institute of Technology, İzmir, Turkey

C. Mitropoulou

ACE Hellas, Athens, Greece

\begin{abstract}
Deployable structures can deploy from a compact to an expanded configuration by changing their sizes. The behaviors of these structures depend on some parameters such as geometric shape, member sizes and kinematic properties. To provide the deployment, not only the arrangements of structural members but also some restrictions must be considered. Moreover, contiguous members of the structures must let the large rotations to provide the transformation between different geometric forms from fully folded to fully deployed configurations. These requirements have an important impact on the fundamental properties of the structures related with structural performance, such as stiffness and strength. In this paper, stiffness of different scissorhinge linkages are analyzed and compared. These linkages cover the same span with almost the same geometry and have the unit elements with same size and same weight. However, the geometry of unit elements is different from each other. The paper investigates the effect of this difference on the stiffness of whole system.
\end{abstract}

\section{INTRODUCTION}

Scissor-hinge linkages are the most preferred type of deployable structures since they are easy to assemble and disassemble. Mostly used in portable or temporary applications such as emergency shelters, exhibition areas, bridges, space enclosures and aerospace applications, these linkages provide significant volume expansion. A typical scissor-hinge linkage system consists of scissor units that are composed of two bars connected by a revolute joint on bars. The linkage is formed by connecting the units to each other with revolute joints at their end nodes.

Deployable structures offer transformability and aesthetic advantages but it is well-known that there is a significant price to pay with respect to their structural response and efficiency. This paper aims to investigate and compare the structural behavior and stiffness of various scissorhinge linkages made up of various primary units, and reveal the best alternative. In order to arrive at this aim, a case study involving six different scissor-hinge linkages are investigated.

\section{STRUCTURAL ANALYSIS}

In this study, different configurations of two linkage types are considered which are transformable scissor-hinge linkage and radial scissor-hinge linkage. The analysis and section optimization is done using Sap2000®. Eurocode 3-2005 provision's strength limit states are used without any load combination to determine the sections of members. Although real life design may be governed by the deflection limit checks, it is not considered in this study since the amount of deflection is an indicator of performance level. The selection of frame section is done among European tubular sections between $60 \times 30 \times 3.6$ and $120 \times 60 \times 4$. In a real life case, the member sections would 
be grouped for manufacturing and assembly purposed. However, to compare the top displacements of the arches at their minimum possible weight would provide a better comparison. Hence, each member is assigned to minimum applicable section (in terms of weight) individually. The sections are assumed to be S275 grade structural steel.

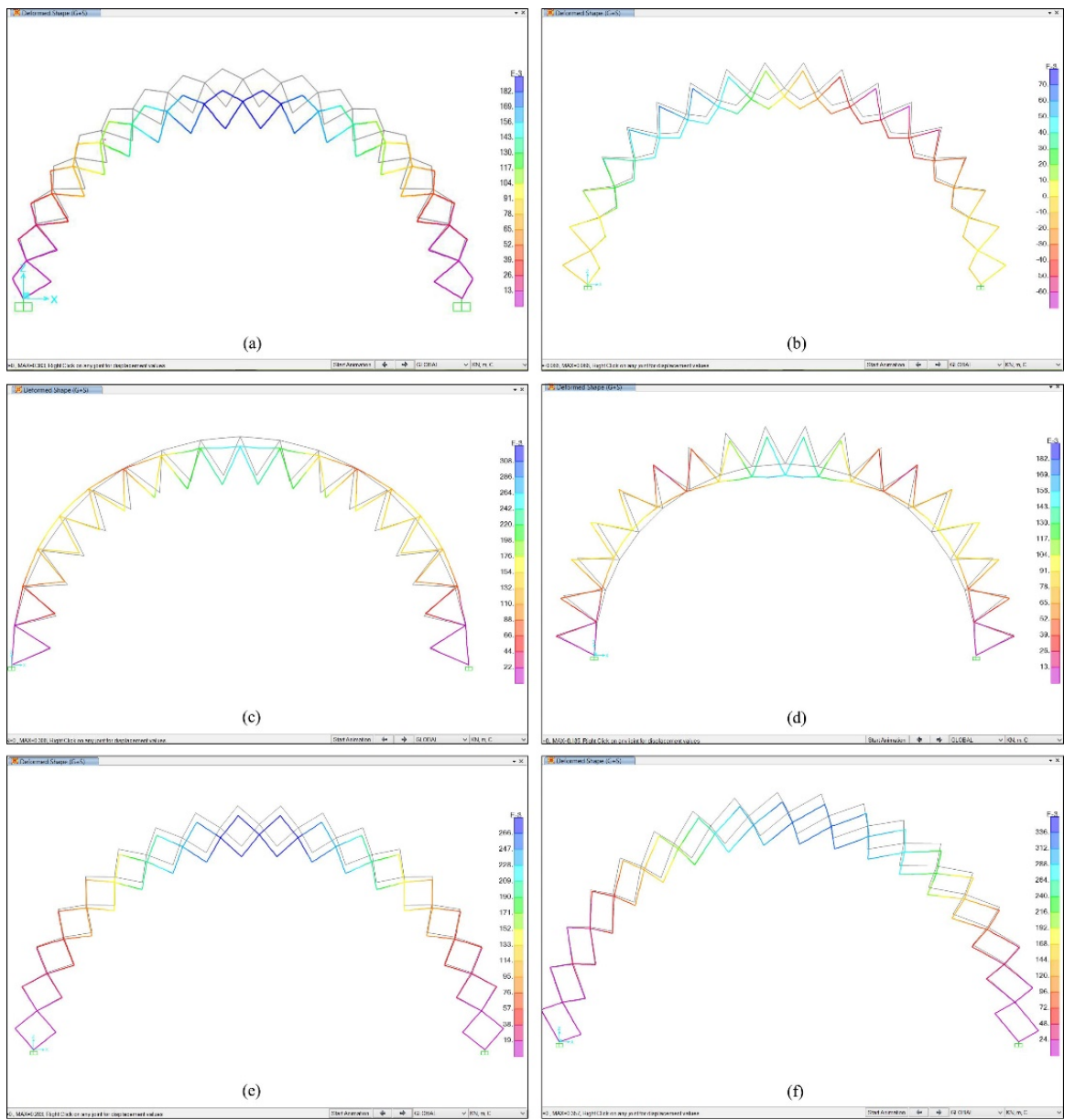

Figure 1. Deformed shapes of scissor-hinge linkages

\section{CONCLUSION}

An initial effort for the quantification of the carrying capacity of scissor-hinge linkages with different primary units has been made. In order to achieve this aim, a case study has been carried out. The scissor-hinge linkages presented in this case study cover the same span with almost the same geometry. Although the size and weight of the unit elements are same, their geometry are different from each other. According to the conducted structural analysis, it has been found that there is a significant difference in stiffness according to the used primary scissor unit. It can be concluded that choosing the proper scissor unit at the initial stage is very important to design a load bearing structure, because the geometric shape of the unit directly affects the structural response of the scissor-hinge linkage.

\section{ACKNOWLEDGMENT}

This work is a part of OptArch project that has received funding from the European Un-ion's Horizon 2020 Research and Innovation programme under the Marie Skłodowska-Curie grant agreement No 689983.

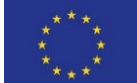

